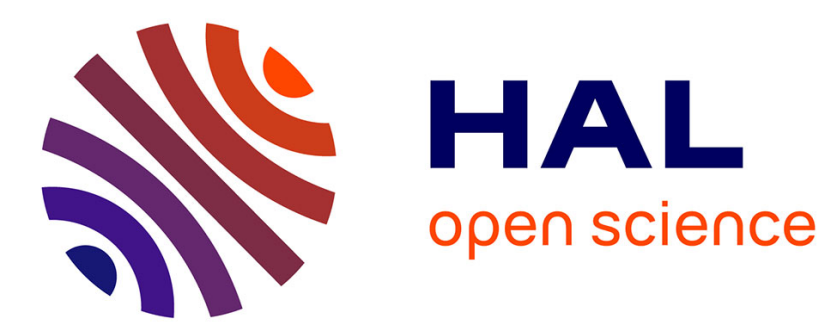

\title{
Le comportement et le concept de choix
}

Jean Baccelli

\section{To cite this version:}

Jean Baccelli. Le comportement et le concept de choix. Dialogue: Canadian Philosophical Review / Revue canadienne de philosophie, 2013, 52 (1), pp.43-60. 10.1017/S0012217313000280 . hal01462277

\section{HAL Id: hal-01462277 \\ https://hal.science/hal-01462277}

Submitted on 14 Feb 2017

HAL is a multi-disciplinary open access archive for the deposit and dissemination of scientific research documents, whether they are published or not. The documents may come from teaching and research institutions in France or abroad, or from public or private research centers.
L'archive ouverte pluridisciplinaire HAL, est destinée au dépôt et à la diffusion de documents scientifiques de niveau recherche, publiés ou non, émanant des établissements d'enseignement et de recherche français ou étrangers, des laboratoires publics ou privés. 


\title{
Le comportement et le concept de choix
}

\author{
JEAN BACCELLI Institut Jean Nicod-École Normale Supérieure, Paris
}

RÉSUMÉ : Cette note examine la partie conceptuelle d'un article de Sen, "Internal Consistency of Choice». Elle isole deux thèses parmi celles que Sen défend. Une première, négative, est qu'aucune condition formelle de cohérence du choix n'est normativement valable sans exception. Une seconde, positive, est qu'une condition formelle de cohérence du choix n'est normativement valable que sous des suppositions faisant intervenir la préférence. Nous procédons à une analyse détaillée des choix énigmatiques dont Sen prend argument et affirmons que cet examen mène à remettre en cause la thèse négative, mais d'une manière subtile qui confirme - même renforcela thèse positive.

ABSTRACT: This note considers the conceptual part of Sen's "Internal Consistency of Choice». Amongst the various claims this paper features, two are singled out. A first, negative, claim is that no formal condition of choice consistency is normatively compelling without exception. A second, positive, claim, is that a formal condition of choice consistency is normatively compelling only under some assumptions involving preference. Here, the puzzling choices Sen puts forward are scrutinized and it is argued that such a scrutiny leads to question the negative claim, but in a subtle way, so that the positive claim is comforted, even strengthened.

\section{Présentation non-technique des termes du problème}

On conçoit ordinairement le choix rationnel comme une décision appropriée étant donné (au moins) deux types de déterminants : des désirs, et des croyances. Par exemple (mais pas seulement), quand elles présentent le choix suivant le critère classique de la maximisation de l'utilité espérée, les théories formalisées 
du choix rationnel ne semblent pas faire table rase d'une telle conception ${ }^{1}$ : l'utilité ramène aux désirs du décideur, et l'on est tenté d'associer l'espérance à ses croyances. Dans une analyse comme dans l'autre, le premier type de cohérence qui caractérise le choix rationnel est une forme (pour ainsi dire verticale) de compatibilité de chaque choix pris isolément avec des désirs et des croyances préalables. Par ailleurs, du moins si plusieurs choix isolés sont réalisés dans les mêmes conditions, une autre forme de cohérence (pour ainsi dire horizontale) peut relier ces choix les uns aux autres.

Les liaisons de ce second type ont été modélisées en grand détail par une littérature spécialisée dans le cadre de théories analysant spécifiquement les formes de choix ${ }^{2}$. Des résultats si remarquables ont été produits, que l'on s'est en substance demandé s'ils n'autorisaient pas l'analyse du choix rationnel à faire abstraction des liaisons du premier type : les liens de cohérence interne entre les choix pourraient être d'une précision telle qu'ils dispensent d'apprécier les liens de cohérence externe pouvant rapporter chaque choix à des désirs et des croyances préalables. Ainsi déterminée, la question a intéressé économistes réflexifs tout autant que philosophes formalistes. Parmi toutes, nourries d'une familiarité particulière avec les données techniques, les analyses de Sen offrent un repère incontournable. À sa manière et à plusieurs reprises, Sen a soutenu que les liens de cohérence externe étaient rigoureusement indispensables à l'appréciation de la rationalité du choix.

Nous examinerons ici l'une de ses contributions à l'étude de ces questions, que l'on lit au début de «Internal Consistency of Choice» ${ }^{3}$ (2004 [1993]). Nous en produisons une critique, avant tout car celle-ci laisse entrevoir un raffinement possible de la position que Sen veut défendre - le titre de cette note laisse entendre que cette critique se veut avant tout une forme d'hommage ${ }^{4}$.

\section{Présentation technique des termes du problème}

Avant toute autre chose, précisons techniquement les termes dans lesquels le problème se pose à Sen, c'est-à-dire : replaçons sa réflexion dans son contexte, celui de la littérature des «préférences révélées». Cette littérature est centrée sur des résultats formels, mais elle est aussi indissociablement marquée par les débats informels que ces résultats ont suscités.

En ce qui concerne les résultats formels, il s'agit de solutions à des problèmes intéressant différentes branches de l'économie. Le plus remarquable d'entre eux est celui dit de l'«intégrabilité». Formulé dans un langage non symbolisé5, le problème consiste à déterminer les propriétés qu'une fonction de demande doit satisfaire pour être obtenue comme la maximisation d'une fonction d'utilité ordinale sous-jacente. De pair avec la solution qui lui a été apportée, en particulier par Samuelson $(1938,1950)$ et Houthakker (1950), ce problème appartient à la théorie micro-économique du consommateur. Mais il s'agit là d'une version spécifique d'un problème général dit de «rationalisabilité» que d'autres théoriciens, comme Arrow (1959) et Sen (1971), ont eux aussi traité : c'est celui qui nous intéressera ici. Formulé dans un langage non symbolisé 6 , le problème est de 
déterminer les propriétés qu'une fonction de choix sur un ensemble quelconque d'options doit satisfaire pour être obtenue comme la maximisation d'une relation de préférence sous-jacente qui soit un pré-ordre total.

Un point est immédiat : le problème général de la «rationalisabilité», formulé en termes ensemblistes, est plus simple que le problème spécifique de «l'intégrabilité», qui fait appel comme son nom l'indique aux techniques du calcul différentiel. Un point n'est pas immédiat : différentes branches de l'économie ont ainsi convergé vers l'étude de problèmes similaires, mais pour des raisons distinctes qui dessinent des enjeux eux aussi distincts. D'un côté, résoudre le problème de l' «intégrabilité» a aidé la micro-économie du consommateur à identifier les conséquences empiriques de sa théorie de l'utilité ordinale. D'un autre côté, résoudre le problème de la «rationalisabilité» a aidé la théorie économique générale ${ }^{7}$ à définir ce qu'est la rationalité du choix précisément conçu, c'est-à-dire la rationalité du choix et non pas celle de la préférence. Dans les deux cas, on doit se rappeler que ces résultats prolongent (et, peut-être, dépassent) les théories traditionnelles du choix rationnel comme maximisation d'une donnée primitive (ici, d'une relation de préférence, là, d'une fonction d'utilité).

Quant aux débats informels, ils mobilisent ces deux problèmes indistinctement. Ils se cristallisent sur une position répandue mais fuyante, qu'on place d'ordinaire sous l'autorité de déclarations méthodologiques faites en marge des contributions techniques. Ainsi Samuelson affirmait-il vouloir «développer une théorie du comportement [...] qui soit débarrassée de toute trace du concept d'utilité» (1938, p. 71). De même Little disait-il espérer «expliquer le comportement en ne se référant à rien d'autre qu'au comportement» $(1949$, p. 90) du moins en ne se référant pas à la préférence ni à l'utilité. Du point de vue historique, il est discuté qu'une telle position méthodologique ait servi de moteur à l'étude des problèmes précédemment mentionnés ${ }^{8}$. Surtout, du point de vue épistémologique, on s'est souvent demandé si une telle position méthodologique, dans la mesure où on peut lui donner un visage précis, est, d'une part, intrinsèquement cohérente, d'autre part, authentiquement liée aux résultats précédemment cités. De telles questions sont au cœur d'une tradition réflexive sur les préférences révélées, toujours active ${ }^{9}$ alors même que les résultats les plus importants sont depuis longtemps parfaitement stabilisés. À l'origine de cette tradition, on trouve un article célèbre de Sen : «Le comportement et le concept de préférence»(1973). En nous concentrant ici sur «La cohérence interne du choix» (2004 [1993]), nous aborderons en fait cette tradition réflexive sous un angle qu'elle n'a pas privilégié : celui de la théorie générale de la rationalité.

\section{Le sujet de Sen et les deux thèses qu'il défend}

S'agissant des résultats formels, Sen se concentre sur le problème de la «rationalisabilité» sous la forme suivante. Soit X un ensemble d'options (pour simplifier, on le supposera fini et contenant au moins trois options). Soit $\mathrm{C}($.) 
une correspondance qui spécifie un «ensemble choisi» non-vide $\mathrm{C}(\mathrm{S}) \subseteq \mathrm{S}$ pour tout «ensemble de choix» ou «menu» $\mathrm{S}$ dans un domaine $\mathrm{K}$ de parties nonvides de $\mathrm{X}$ (pour simplifier, on supposera que $\mathrm{K}$ contient au moins tous les singletons, paires et triplets d'options de $\mathrm{X}$ ). Définissons une relation $\mathrm{R}^{*}$ que l'on nommera «relation de préférence révélée» $: \forall \mathrm{x}, \mathrm{y} \in \mathrm{X}, \mathrm{xR} * \mathrm{y}$ ssi $\exists \mathrm{S} \in \mathrm{K}$ t.q. $\mathrm{x} \in \mathrm{C}(\mathrm{S})$ et $\mathrm{y} \in \mathrm{S}$ - insistant sur le fait que $\mathrm{R}^{*} \mathrm{n}$ 'est qu'une abréviation pour un choix remplissant ces conditions. Le problème est d'identifier les propriétés auxquelles $\mathrm{C}($.) doit satisfaire pour que se vérifie :

$$
\forall \mathrm{S} \in \mathrm{K}, \mathrm{C}(\mathrm{S})=\left\{\mathrm{x}: \mathrm{x} \in \mathrm{S} \text { et } \forall \mathrm{y} \in \mathrm{S}, \mathrm{xR}^{*} \mathrm{y}\right\}
$$

c'est-à-dire pour que l'on puisse «rationaliser» la correspondance de choix $\mathrm{C}$ par la relation de préférence révélée $\mathrm{R}^{*}$. Ces propriétés, dites «inter-menus» car elles portent sur les liens entre différents choix faits depuis différents menus, varieront selon que l'on cherche à rationaliser la correspondance par une relation complète, complète et quasi-transitive, ou complète et transitive : on retrouve ainsi, sans se prononcer sur le fond, la forme de la théorie traditionnelle du choix rationnel provenant d'une relation de préférence. Les propriétés recherchées sont bien connues ${ }^{10}$. Est dans tous les cas requis le respect d'une propriété, nommée «propriété $\alpha$, qui est satisfaite ssi :

$$
\forall \mathrm{S} \subseteq \mathrm{T} \in \mathrm{K} \text {, si } \mathrm{x} \in \mathrm{C}(\mathrm{T}) \text { et } \mathrm{x} \in \mathrm{S} \text {, alors } \mathrm{x} \in \mathrm{C}(\mathrm{S})^{11}
$$

(si une pomme est choisie entre \{une pomme, une poire, une orange\}, une pomme est aussi choisie entre \{une pomme, une poire\}). Techniquement, cette forme de cohérence dans la contraction est la plus faible des propriétés classiques des fonctions de choix. Par ailleurs, normativement, elle semble assez irrésistible.

S'agissant des débats informels, Sen se concentre sur la thèse selon laquelle des propriétés de cohérence devraient être considérées comme «interne[s] au choix» (2004 [1993], p. 121). Parlant de «cohérence interne» du choix, Sen désigne parfois, de manière neutre, des formes de correspondance interne au domaine des menus, comme la propriété $\alpha$, mais il désigne ainsi surtout, de manière critique, la position suivant laquelle on pourrait par elles requérir «des liens entre différentes parties d'une fonction de choix sans se référer à quoi que ce soit d'extérieur au choix (comme des motivations, des objectifs, des principes)» (ibid., p. 122). Certes, la détermination de telles références extérieures - motivations, objectifs, principes - reste vague, mais elle ne l'est cependant pas assez pour que l'on ne puisse y reconnaître, par distinction avec la croyance, des traces de la préférence. C'est en tout cas ainsi que nous lirons ici $\operatorname{Sen}^{12}$, rattachant de la sorte explicitement sa cible à la position fuyante dont nous avons dit qu'elle cristallisait les débats informels sur les préférences révélées. Cette position prend alors la forme de la thèse suivant laquelle les correspondances inter-menus classiques ne caractérisent pas les implications pour le choix 
d'une préférence vérifiant telle ou telle condition de rationalité : déliées de toute référence à la préférence, elles caractérisent la rationalité du choix tout court, qui n'est affaire que de telles correspondances inter-menus ${ }^{13}$.

Dans la partie conceptuelle de son article - mettant ainsi en cause non pas le contenu, mais un «usage» (ibid., p. 124, n. 4) des propriétés classiques des fonctions de choix -, Sen développe une critique de cette position de la «cohérence interne» du choix. On peut la préciser en distinguant en elle, un peu plus fermement que Sen ne le fait ${ }^{14}$, deux thèses.

Une thèse que nous appellerons «négative» nie que l'on puisse «imposer» (ibid., p. 122) inconditionnellement à une fonction de choix le respect de quelque propriété que ce soit ${ }^{15}$, ce que Sen exprime comme suit (c'est lui qui souligne) : aucune forme de «correspondance interne» n'a le statut d'un réquisit inconditionnel de «cohérence interne» (ibid., p. 122). En d'autres termes, aucune condition formelle de cohérence du choix n'est normativement valable sans exception - thèse négative qui est aussi (logiquement) simple, au sens de n'être pas composite. La défense de cette thèse mène Sen à étudier, à titre d'exemples censés emporter l'adhésion, des cas de choix énigmatiques dont il entend établir la «cohérence interne» alors même qu'ils ne semblent respecter pas même la forme de «correspondance interne» spécifiée par la propriété $\alpha^{16}$ - pourtant la plus faible de toutes les propriétés classiques des fonctions de choix.

Une thèse que nous appellerons «positive» affirme que pour décider si une fonction de choix est cohérente ou incohérente (en un sens qui ait à voir avec la rationalité), il faut «de toute façon aller au-delà des caractéristiques internes d'une fonction de choix» (ibid., p. 124), suivant des références qui lui sont extérieures et qui - comme il a été spécifié - impliquent la préférence. En d'autres termes, une condition formelle de cohérence du choix n'est normativement valable que sous des présuppositions faisant intervenir la préférence. Notons que la thèse positive est (logiquement) complexe, recouvrant deux sous-thèses : la cohérence du choix s'apprécie d'une manière non pas interne, mais externe; pour être plus spécifique, c'est relativement à la préférence qu'elle s'apprécie. Notons aussi que les liens (logiques) unissant thèse négative et thèse positive sont plus fins qu'ils ne pourraient d'abord sembler l'être. Certes, la première pourrait se trouver éclairée par la seconde, de la manière suivante : peut-être aucune condition formelle de cohérence du choix n'est-elle normativement valable sans exception, car suivant la manière dont la préférence jouera, des cas de figure exceptionnels pourront apparaître. Pour autant, la thèse négative n'est pas impliquée par la thèse positive, car, dans sa simplicité (logique), elle n'entre pas dans la distinction de l'interne et de l'externe, se contentant de dire qu'à toute condition formelle de cohérence du choix, on peut opposer un contre-exemple normativement défendable. Or, il n'en est pas moins possible que sous certaines suppositions impliquant la préférence, il existe une condition formelle de cohérence du choix qui se révèle normativement valable d'une manière universelle. 
Nous défendrons que c'est cette ligne, subtile mais importante, qu'invite à tenter un examen scrupuleux des cas de choix énigmatiques sur lesquels Sen fonde l'essentiel de son argumentation. Ceux-ci sont devenus célèbres, si bien qu'il existe à leur sujet une littérature technique spécialisée, dont on trouvera une revue, par exemple, dans Bossert et Suzumura (2011). Cela reconnu, il faut préciser que cette littérature a moins pour habitude de disséquer ces cas de choix énigmatiques, que de les verser tels quels à ses discussions, pour se demander surtout si, pris pour argent comptant, ils invitent à une généralisation des propriétés standards des fonctions de choix (le cas échéant, à laquelle) ou à une exclusion de certaines complications de leur domaine d'application (le cas échéant, desquelles et pourquoi). Nous montrerons pourtant, avant tout, le caractère fructueux d'un examen critique de ces cas.

\section{Examen critique des cas de choix énigmatiques}

Les cas de choix énigmatiques que nous étudions suivent tous le même schéma :

$$
\text { (1) }\{\mathrm{y}\}=\mathrm{C}(\{\mathrm{x}, \mathrm{y}, \mathrm{z}\}) \text { et }(2)\{\mathrm{x}\}=\mathrm{C}(\{\mathrm{x}, \mathrm{y}\})
$$

Toute fonction de choix instanciant ce schéma viole la propriété $\alpha^{17}$. En avançant des interprétations rationnelles pour la conjonction ((1) et (2)), Sen entend étayer les thèses négative et positive d'un même mouvement. Nous discutons les trois principales interprétations tour à tour, tâchant de les expliciter au mieux : les cas ont un intérêt propre pour l'esprit, indépendamment du débat dans lequel Sen veut les faire jouer.

Un premier exemple porte sur le cas d'un ascète sourcilleux. Sen écrit : «le sens du jeûne, sous la forme de ne pas manger (y), étant donnée la possibilité de bien manger (z), peut devenir moins clair quand la seule autre option est d'être en partie affamé (x)» (2004 [1993], p. 131). Pour faire valoir ce cas dans le cadre du débat sur la rationalité de certaines violations de la propriété $\alpha$, il faut faire abstraction de deux aspects à cette fin superflus ${ }^{18}$. Il faut d'abord faire abstraction de problèmes d'observation : l'exemple n'aurait que peu de portée s'il servait à dire qu'un observateur extérieur, en l'absence d'assez de nourriture, ne saurait distinguer le choix du jeûne du choix de la famine. Mais il faut aussi faire abstraction de problèmes de définition : l'exemple n'aurait que peu de portée s'il servait à dire qu'en l'absence d'assez de nourriture, le choix du jeûne ne serait tout simplement pas possible (les menus d'options seraient alors non pas $\{x, y, z\}$ et $\{x, y\}$, mais $\{x, y, z\}$ et $\{x\}$, si bien que sous l'hypothèse de (1) la propriété $\alpha$ ne serait en fait tout simplement pas mise à l'épreuve, puisque bien entendu $\mathrm{y} \notin\{\mathrm{x}\}$ ).

Dans un autre article, Sen présente un exemple proche mais moins équivoque relativement à ces deux aspects (2004 [1997], p. 172, n. 25); nous l'utiliserons donc pour comprendre notre cas. Dans un régime autoritaire, esquisse-t-il, un lecteur libéral pourrait choisir de lire le journal officiel (x), s'il a le choix entre le lire, ne rien lire (y) et lire un journal dissident (z), et pourtant choisir de ne 
rien lire plutôt que de lire le journal officiel, si la possibilité de lire le journal dissident n'existe plus : il ne veut pas que l'on choisisse pour lui. Par analogie, le cas de l'ascète sourcilleux se comprendrait comme suit. S'il y a assez de nourriture, l'ascète choisit de s'en dispenser; mais il veut être l'origine de son choix et s'il n'y en a pas assez, il choisit de manger le peu de nourriture qu'il y a. Sen parle à ce sujet d'une «liberté de rejeter» ${ }^{19}$ (2004 [1993], p. 131), dont les conditions ne seraient plus, dans le second cas, réunies.

En fait, il semble qu'on doive dès lors prêter à l'ascète sourcilleux une fonction de choix définie à l'échelle d'un menu de menus ${ }^{20}$. Son souci d'être celui qui choisit de jeûner implique que face à $\{\{x, y, z\},\{x, y\}\}$, son choix vérifie (3) $\{\mathrm{x}, \mathrm{y}\} \neq \mathrm{C}(\{\{\mathrm{x}, \mathrm{y}, \mathrm{z}\},\{\mathrm{x}, \mathrm{y}\}\})=\{\mathrm{x}, \mathrm{y}, \mathrm{z}\}$. D'ailleurs, sans doute rejetterait-il $\{x, y\}$ dès que possible : par exemple, si w symbolisait la présence de beaucoup de nourriture, (4) $\{\mathrm{x}, \mathrm{y}\} \neq \mathrm{C}(\{\{\mathrm{w}, \mathrm{x}, \mathrm{y}, \mathrm{z}\},\{\mathrm{x}, \mathrm{y}, \mathrm{z}\},\{\mathrm{x}, \mathrm{y}\}\})$ serait plausible. Un tel choix de haut niveau n'a rien d'énigmatique — en tout cas, la conjonction ((3) et (4)) respecte bien, elle, la propriété $\alpha$. L'énigme du choix de plus bas niveau, exprimée par la conjonction de ((1) et (2)), provient manifestement du fait que l'interprétation investit ici le concept de choix d'un sens intentionnel particulièrement fort. Mais c'est précisément cette charge intentionnelle que capture à sa manière la description du choix à l'échelle des menus : loin d'être une redescription arbitraire, celle-ci met en valeur l'objet fondamental du choix de l'ascète sourcilleux. Par ailleurs, et pour terminer sur ce cas, notons que quoique discrètement présente, la teneur stratégique des choix examinés ne semble pas ici cruciale ${ }^{21}$ : on peut certes lire (2) comme l'adresse d'une sorte de protestation, mais le problème est entier même si l'on décrit la situation, comme nous l'avons fait ici, sans spécifier aucune interaction particulière.

Un deuxième exemple porte sur le cas d'un mondain soupçonneux. Sen écrit : «quelqu'un qui, confronté au choix entre aller prendre le thé chez une vague connaissance $(\mathrm{x})$ et ne pas y aller $(\mathrm{y})$, choisit d'aller prendre le thé $(\mathrm{x})$, n'en pourrait pas moins choisir de ne pas y aller (y) si cette même connaissance lui offre le choix entre aller prendre le thé (x), ne pas y aller (y) et prendre un peu de cocaïne (z)» (2004 [1993], p. 130).

Clairement, on a là un descendant d'un plus célèbre gourmet soupçonneux imaginé d'après ${ }^{22}$ Luce et Raiffa $(1957$, p. 288). Devant choisir dans un restaurant où il n'est jamais allé entre du poisson à la vapeur $(\mathrm{x})$ et un steak tartare $(\mathrm{y})$, le gourmet, soupçonneux, choisit le poisson à la vapeur. Mais, rassuré, il choisit le steak tartare si on lui précise que le menu propose aussi des cuisses de grenouilles (z) : c'est à ses yeux le signe d'un restaurant de qualité qui saura lui servir un bon steak tartare. La structure de l'exemple de Sen est proche. S'il n'y a pas de cocaïne, le mondain pense qu'il dégustera son thé en bonne compagnie et choisit donc d'accepter l'invitation; mais s'il y a de la cocaïne, il craint de déguster son thé en mauvaise compagnie et choisit donc de la refuser. Il était incertain ${ }^{23}$ quant à la qualité de la compagnie de cette connaissance et le menu le plus large a levé son incertitude. Sen parle à ce sujet de «valeur épistémique du menu» (2004 [1993], p. 130). Ce caractère épistémique, cela dit, ne doit pas faire perdre de vue le point 
central : le décideur a par hypothèse une attitude préférentielle (le peu d'appétence pour la cocaïnomanie) qui se nourrit de l'information extraite des menus.

En ce qui concerne le débat sur la rationalité de certaines violations de la propriété $\alpha$, il nous semble que ce cas est limité, dans la mesure où l'ordre suivant lequel les menus sont présentés paraît crucial. En effet, si le décideur est confronté au menu $\{\mathrm{x}, \mathrm{y}\}$, puis au menu $\{\mathrm{x}, \mathrm{y}, \mathrm{z}\}$, alors on pourra avoir une conjonction comme ((1) et (2)). Mais si le décideur est confronté au menu $\{\mathrm{x}, \mathrm{y}, \mathrm{z}\}$, puis au menu $\{\mathrm{x}, \mathrm{y}\}$, on aura plutôt une conjonction comme ((1) et (5)), avec (5) $\{\mathrm{y}\}=\mathrm{C}(\{\mathrm{x}, \mathrm{y}\})$ : sans doute, aux yeux du mondain, un cocaïnomane même sans cocaïne resterait-il de mauvaise compagnie. Mais alors, aucune violation de la propriété $\alpha$ n'aurait lieu. Il en irait de même si on lui présentait les deux menus à la fois ou si on lui accordait la possibilité de réviser un choix déjà fait à la lumière de l'information extraite d'un autre choix à faire.

Or, de manière générale, le domaine classique des fonctions de choix est un ensemble — pas une liste séquentielle — de menus, tout effet d'ordre étant donc hors sujet. Cet aspect purement technique peut faire l'objet d'une interprétation conceptuelle : on s'intéresse aux choix du décideur en équilibre réfléchi. De la sorte, le problème n'est pas tant qu'il y ait une information dépendante aux menus à laquelle le décideur est sensible; il est plutôt que la totalité de l'information dépendante aux menus ne soit pas extraite une fois pour toutes ${ }^{24}$. Il ne s'agit pas de sous-estimer la valeur épistémique des menus, mais de l'apprécier dans les conditions du problème initial, qui ne permettent pas de la faire ressortir comme Sen le veut. À notre connaissance, malgré sa simplicité ${ }^{25}$, la critique n'a pas été produite par la littérature.

Un troisième exemple porte sur le cas d'un amateur de fruits courtois. Sen suggère en effet (2004 [1993], p. 129) que l'on pourrait, par courtoisie, choisir de ne rien prendre (x) plutôt que de prendre la pomme (y) d'un panier qui n'en contient pas d'autre, mais choisir cette même pomme (y) si une autre (z) était ajoutée au panier (ne rien prendre étant toujours une option). Ce cas paraît particulièrement pur. Il n'est parasité par aucun problème d'observation ou de définition, ni limité par aucune sensibilité à l'ordre de présentation des menus. Par ailleurs, sa logique est particulièrement claire : s'il y a assez de fruits d'un certain type, alors l'amateur de fruits en prendra; sinon, fidèle à la courtoisie qu'il s'impose, il s'en abstiendra. Sen parle à ce sujet, dans des termes qui lui sont propres, de «choix positionnel» (ibid., p. 130).

Ici, la propriété $\alpha$ ne paraît violée que ponctuellement : dès que deux fruits d'un même type, ou plus, seront présents, elle pourra, semble-t-il, être uniformément respectée ${ }^{26}$. Mais de telles règles de courtoisie peuvent engendrer des violations plus systématiques encore de la propriété. Étendant une variation de Sen (ibid., p. 130) sur le cas précédent, on imaginera par exemple un gourmand courtois qui aurait comme règle de prendre la part de gâteau qui soit juste en-dessous, en taille, de la plus grosse disponible. Il présenterait une fonction de choix qui violerait non pas une, mais plusieurs fois, la propriété $\alpha$. Interprétant $\mathrm{x}^{0}, \mathrm{x}^{1}, \ldots, \mathrm{x}^{\mathrm{n}}$ comme des parts de taille croissante, la propriété $\alpha$ serait violée 
de manière répétée dans la série suivante : (7) $\left\{\mathrm{x}^{0}\right\}=\mathrm{C}\left(\left\{\mathrm{x}^{0}, \mathrm{x}^{1}\right\}\right),(8)\left\{\mathrm{x}^{1}\right\}=$ $\mathrm{C}\left(\left\{\mathrm{x}^{0}, \mathrm{x}^{1}, \mathrm{x}^{2}\right\}\right), \ldots,(9)\left\{\mathrm{x}^{\mathrm{n}-1}\right\}=\mathrm{C}\left(\left\{\mathrm{x}^{0}, \ldots, \mathrm{x}^{\mathrm{n}}\right\}\right)$. Ainsi, la propriété $\alpha$ semble passer à côté d'une forme de cohérence pourtant particulièrement saillante.

Mais, si l'on s'intéresse directement aux différentes tailles disponibles, les présentant en ordre décroissant $\mathrm{t}^{1}, \ldots, \mathrm{t}^{\mathrm{n}}$, on aura $(10)\left\{\mathrm{t}^{2}\right\}=\mathrm{C}\left(\left\{\mathrm{t}^{1}, \mathrm{t}^{2}\right\}\right)=\ldots=$ $\mathrm{C}\left(\left\{\mathrm{t}^{1}, \mathrm{t}^{2}, \ldots, \mathrm{t}^{\mathrm{n}}\right\}\right)^{27}$, ce qui rendra manifeste un respect de la propriété $\alpha$ que la description strictement physique des options ne permettait pas de discerner. Le respect de la propriété $\alpha$ n'est pas ainsi trivial, au sens où la propriété est bien mise à l'épreuve. Par ailleurs, la motivation de la redescription n'est pas de sauver la validité de la propriété, mais d'intégrer la manière dont le décideur se représente ${ }^{28}$ les options. Même si la structure à exploiter est alors moins apparente, on peut jusqu'à un certain point développer une analyse analogue du cas de l'amateur de fruits courtois. La description a introduit (y) comme la première pomme, mais dans $\{\mathrm{x}, \mathrm{y}, \mathrm{z}\}$, il ne s'agit pas moins d'une deuxième pomme; or dans ces termes, qui sont ceux de la délibération de l'amateur de fruits courtois, son choix respectera la propriété $\alpha$.

Notons enfin que ces deux cas cousins ont une teneur stratégique indéniable ${ }^{29}$. Or celle-ci est ambivalente dans la mesure où les exemples sont compatibles avec différents jeux qui illustrent tant le respect de la propriété $\alpha$ que sa violation — que l'inexistence pure et simple d'une fonction de choix sur les menus d'options. En effet, il semble nécessaire d'expliciter au moins un autre «joueur» vis-à-vis duquel l'amateur de fruits, par exemple, peut se comporter de manière courtoise : nommons-le «l'hôte» et supposons qu'il est le seul autre joueur d'un «jeu du fruit» ${ }^{30}$. Quels que soient les détails exacts de l'arbre de ce jeu, si l'hôte a déjà été servi, la fonction de choix de l'amateur de fruits pourra alors sans discourtoisie vérifier à la fois $(1)\{\mathrm{y}\}=\mathrm{C}(\{\mathrm{x}, \mathrm{y}, \mathrm{z}\})$ et $(5)\{\mathrm{y}\}=\mathrm{C}(\{\mathrm{x}, \mathrm{y}\})$, et la propriété $\alpha$ sera respectée. En revanche, si l'hôte n'a pas déjà été servi, la fonction de choix de l'amateur de fruits courtois devra vérifier (1) $\{\mathrm{y}\}=\mathrm{C}(\{\mathrm{x}, \mathrm{y}, \mathrm{z}\})$ et (2) $\{x\}=C(\{x, y\})$, et la propriété $\alpha$ sera violée. Mais dès lors, si un jeu du fruit est assez complexe pour que l'arbre explore les deux possibilités, sa fonction de choix au sens qui nous intéresse vérifiera respectivement, aux nœuds de choix pertinents de l'arbre, (5) $\{\mathrm{y}\}=\mathrm{C}(\{\mathrm{x}, \mathrm{y}\})$ et (2) $\{\mathrm{x}\}=\mathrm{C}(\{\mathrm{x}, \mathrm{y}\})^{31}$. À proprement parler, il n'existera donc pas pour lui de fonction de choix sur les menus d'options. Sous les mêmes prémisses, Sen pourrait donc aisément pousser plus loin son objection : certains choix ne semblant pas même définir une fonction de choix peuvent en réalité se révéler être cohérents. L'objection invite d'autant plus à se demander si la fonction de choix n'est pas mal définie (au lieu d'être non définie), ce qui revient à souligner qu'il serait étrange d'accorder la possibilité ici, mais de la refuser ailleurs.

\section{5. Évaluation des thèses}

Que conclure de l'examen des cas énigmatiques en ce qui concerne les deux thèses identifiées dans la critique par Sen de la position de la «cohérence interne» du choix? 
Il nous semble que la thèse négative n'en ressort pas renforcée, dans la mesure où la portée de la propriété $\alpha$ résiste bien mieux que prévu aux cas qui lui sont opposés. L'examen critique du second exemple autorise la conclusion la plus nette. C'est une fois tous les menus d'options donnés qu'il faut définir la fonction de choix. Quand on procède ainsi, les violations rationnellement défendables de la propriété $\alpha$ ciblées par l'exemple disparaissent. Le premier exemple appelle, lui, un diagnostic plus subtil. Sen considère la liberté de rejeter une option depuis un menu. Mais celle-ci renvoie à la liberté de choisir un menu depuis lequel on puisse ainsi rejeter l'option. Or, un tel choix des menus d'options, qui est l'aspect déterminant de l'exemple, valide bien, en revanche, la propriété $\alpha$. Le troisième exemple est le plus intéressant de tous et il invite à une appréciation nuancée. Il faut se concentrer sur les spécifications d'un contexte stratégique qui mènent (ce qui n'est pas toujours le cas) à des violations par la fonction de choix de la propriété $\alpha$. Même dans ces cas, il reste qu'une description de la fonction de choix fidèle à la manière dont, par hypothèse, le décideur traite les options valide bien, elle, la propriété $\alpha$. Certes, ces redescriptions des premier et troisième exemples n'annulent pas totalement les violations présentées par les fonctions d'origine; mais elles en limitent la portée pour le sujet de la thèse négative strictement délimitée.

En fait, l'examen critique des cas énigmatiques suggère ainsi un raffinement $^{32}$ de la thèse négative de Sen, que celui-ci ne considère pas. Sen pourrait en effet avancer que sans information sur le contexte du choix (information dont il affirme par ailleurs la nature préférentielle, c'est là sa thèse positive que nous évaluons plus bas), on ne peut pas préciser sur quelles entités exactement un choix doté de cohérence interne va manifester son respect de la correspondance interne spécifiée par la propriété $\alpha$. Certes, il peut tout à fait s'agir des options simplement données; mais il s'agira parfois d'objets autrement plus complexes. L'examen des exemples semble en tout cas compatible avec la proposition suivante ${ }^{33}$ : si un choix est cohérent, alors on peut être assuré $d u$ fait qu'il respecte la propriété $\alpha$, sans pour autant savoir la manière dont ce respect apparaîtra. Comme nous allons le voir, la thèse positive de Sen éclaire ainsi le sujet de sa thèse négative.

Pour ce qui est de la thèse positive, il nous semble qu'elle ressort renforcée de l'examen des cas. Nous le justifions en considérant les deux composantes de la thèse, désormais telles que Sen les présente. La première avance que pour apprécier la cohérence ou l'incohérence d'une fonction de choix, il faut préciser un contexte pour le choix. Sen écrit ainsi : «une fonction de choix n'est pas cohérente ou incohérente sans interprétation - sans une idée sur le contexte [...]» (2004 [1993], p. 127). La deuxième avance que l'information cruciale fournie par le contexte relève, comme nous l'avons justifié plus haut, de la préférence ${ }^{34}$. Sen écrit ainsi : «le contexte [...] nous emmène au-delà de la fonction de choix — vers des motivations, des objectifs, des principes» (ibid., p. 127, n. 10). Les exemples donnent indiscutablement crédit à la première composante de la thèse - mais elle est assez consensuelle, en tout cas si elle 
désigne la nécessité d'une interprétation (au sens méta-logique). Cela posé, donnent-ils de quoi résister à la deuxième composante de la thèse, qui l'est moins? Pour comprendre que tel n'est pas le cas, il suffit d'imaginer pour chacun de nos décideurs un jumeau doté de la même fonction de choix d'origine, des mêmes croyances initiales implicites - mais non sourcilleux, non soupçonneux ou non courtois. Ce serait ainsi une variation exclusivement préférentielle qui rendrait cohérent le choix de l'ascète sourcilleux et incohérent celui de son jumeau qui voudrait simplement ne rien manger; cohérent le choix du mondain soupçonneux et incohérent celui de son jumeau qui n'accorderait aucune importance à la compagnie de son hôte ${ }^{35}$; cohérent le choix de l'amateur de fruit courtois et incohérent celui de son jumeau faisant fi de toute convenance. Finalement, ils ne concevraient tout simplement pas leurs choix dans les mêmes termes.

Un aspect dialectique devient ainsi clair. Suivant l'analyse précédente, on comprendra que la discussion des cas de choix semblant violer la propriété $\alpha$ forme un piège pour un partisan forcené de la position de la cohérence interne du choix, en particulier s'il espère arguer de la généralité des résultats formels de la littérature des préférences révélées pour développer quelque thèse forte que ce soit sur la préférence. D’un côté, la généralité de la validité de la propriété $\alpha$ doit être préservée : elle est nécessaire pour tous les résultats dont on peut vouloir se servir. D'un autre côté, elle ne peut être préservée qu'au prix d'analyses qui reviennent à concéder la nécessité, pour apprécier la rationalité d'un choix, de se référer à la préférence.

En réalité, la discussion s'est constamment appuyée sur des attitudes préférentielles implicites qui contribuent jusqu'à l'individuation des options entre lesquelles il est en question que tel choix soit cohérent. Certes, l'approche intensionnelle a toujours un prix, que certaines méthodologies peuvent par principe se refuser à payer. Mais, d'une part, elle a ici l'avantage de faire ressortir la généralité de certaines propriétés du choix rationnel, au lieu que celles-ci soient fragmentées en plusieurs généralisations. D’autre part et surtout, la leçon subtile des exemples est que cette approche est inévitable. En fait, le cadre du problème de la rationalisabilité, portant sur un domaine quelconque, rend saillant le point suivant : «la première étape est [...] la spécification de l'ensemble général des options, qui va directement résulter de nos idées concernant ce qui compte vraiment pour l'agent» (Bhattacharyya et al., 2011, section 6). Le débat sur la portée des propriétés classiques des fonctions de choix n'est qu'une occasion indirecte de mettre cette intrication fondamentale en pleine lumière.

C'est pour la thèse positive de Sen une précision, qu'il ne considère pas non plus. En fait, la thèse pourrait s'entendre en un sens faible : une condition formelle de cohérence du choix n'est normativement valable que sous des présuppositions faisant intervenir une préférence, laquelle n'intervient cependant pas dans l'individuation des objets du choix (par exemple, on dira que pour savoir comment individuer de tels objets, il suffit de connaître les croyances du décideur). 
Mais elle pourrait aussi s'entendre en un sens fort : une condition formelle de cohérence du choix n'est normativement valable que sous des présuppositions faisant intervenir une préférence, laquelle intervient jusque dans l'individuation des objets du choix (par exemple, on dira que pour savoir comment individuer de tels objets et pouvoir apprécier le travail des croyances du décideur, il faut d'abord connaître quelque chose de ses préférences). La variation sur les décideurs et leurs jumeaux éclaire aussi ce sujet : c'est visiblement la version forte que les cas de choix énigmatiques ici étudiés favorisent.

\section{Conclusion}

On peut estimer qu'il y a chez Sen, de son article fondateur de 1973 à celui de 1993 sur lequel nous nous sommes concentrés ici, un resserrement dans la réflexion critique sur la littérature des préférences révélées. De fait, nourrie de problèmes plus intérieurs à la théorie du choix, la réflexion se développe désormais depuis l'angle du choix, plutôt que depuis celui de la préférence. Ce n'est pas seulement la préférence que l'on risque de mal concevoir en lisant trop vite les résultats formels de la littérature : c'est, avant elle, le choix lui-même. Pour comprendre le choix, on ne peut se dispenser ${ }^{36}$ de considérer la préférence et analysant celui-ci, on ne se débarrasse pas de celle-là : on tend à mieux en identifier les rôles.

Or, que Sen l'ait souhaité ou non, ces rôles mènent à interroger la possibilité non pas d'une, mais de deux réductions assez distinctes : celle de la rationalité du choix à des propriétés inter-menus des fonctions de choix et celle de l'individuation des options de choix à une simple donnée extensionnelle. C'est ce que suggère l'analyse détaillée des cas de choix énigmatiques que Sen signale, puisqu'elle inspire une thèse intermédiaire entre celles qu'il défend. Il s'agit de concéder entièrement la thèse positive, tout en restant réservé s'agissant de la thèse négative : il est frappant que, quoique sur des objets d'une complexité variable, le choix rationnel semble toujours manifester un respect non trivial de ladite «propriété $\alpha$ ».

De manière plus générale, ce resserrement dans la réflexion critique sur la littérature des préférences révélées — qui marque un progrès dans l'examen suggère que l'on peut adopter une lecture à contre-courant des débats informels qu'elle contient : il s'agit de se focaliser moins sur les mauvaises analyses du concept de préférence, que sur les bonnes analyses du concept de choix que ces débats peuvent receler. D'où le titre qu'en référence à l'article fondateur de Sen nous avons donné à cette note : «Le comportement et le concept de choix» ${ }^{37}$.

\section{Notes}

1 Ce n'est là qu'une apparence, que des épistémologues de la théorie de la décision ont pu vouloir dissiper pour différentes raisons (cf. par exemple Mongin, 2011 et Pettit, 1991 pour des éclairages distincts sur le problème). 
2 Nous pensons à la littérature des «préférences révélées» dont la présentation fera l'objet de la section suivante : après les préalables conceptuels qui occupent cette section, celle-ci sera consacrée à des préalables techniques.

3 Nous réfèrerons désormais à cet article sous le titre français : «La cohérence interne du choix». Nous proposons ainsi notre traduction, comme partout ailleurs dans cette note dès que cela sera nécessaire.

4 En effet, le premier texte que Sen a consacré à la question a pour titre éloquent : «Behaviour and the Concept of Preference», c'est-à-dire «Le comportement et le concept de préférence» (1973). L'inspiration est si transparente qu'elle n'appelle pas ici plus de commentaire.

5 Pour un énoncé formel standard, cf. MasCollel et al. (1995), section 3.H (la section 2.E rappelle ce qu'est une «fonction de demande»). On réfère ainsi au problème global en utilisant le nom de sa partie la plus difficile.

6 Pour un énoncé formel standard, cf. MasCollel et al. (1995), section 1.D. Nous détaillons ce problème plus loin.

7 Ce fut en particulier le cas pour la théorie du choix social, fondée notamment par Arrow et Sen. À un stade formateur où ses objets n'avaient pas de structure particulière, le problème de la rationalisabilité l'a aidée à donner un sens formel à ce concept de «choix» social ainsi qu'à celui, qui peut lui être lié, de «préférence» sociale. Par ailleurs, le contexte de la théorie du choix social définit pour les débats informels qui nous occuperont des enjeux propres, souvent sous-estimés. Même s'ils affleurent chez Sen, nous les ignorerons ici.

8 On estime d'ordinaire que tel est le cas. Cf. Wong (2006 [1978]) pour une étude des travaux pionniers de Samuelson explicitement menée depuis ce présupposé, et Mongin (2000) pour une critique explicite du présupposé.

9 Cf. Hausman (2000) pour un exemple de développements récents du pan critique de la tradition. Par des voies complexes, l'essor récent de la «neuroéconomie» a mené les partisans de la position méthodologique en question à en rendre plus virulente encore la formulation : cf. par exemple Gul et Pesendorfer (2008).

10 Cf. Sen (1971), respectivement sections 9, 10 et 8.

11 D'une manière équivalente, mais plus élégante, on pourra l'exprimer de la façon suivante :

$$
\forall \mathrm{X}, \mathrm{Y} \in \mathrm{K}: \mathrm{C}(\mathrm{X} \cup \mathrm{Y}) \subseteq[\mathrm{C}(\mathrm{X}) \cup \mathrm{C}(\mathrm{Y})]
$$

12 C'est une lecture simplificatrice mais non arbitraire. Résumant sa critique (2004 [1993], p. 132), Sen se réfère à Davidson et à sa catégorie générale de «pro-attitude», assez proche de celle de «préférence». Nous n'exploiterons pas ici le fait que le grain conatif de ces motivations, objectifs, principes n'est clairement pas quelconque. Cela dit, sans doute Sen serait-il aussi sensible à ce qui peut séparer ces motivations, objectifs, principes, qu'à ce qui les réunit : c'est ce que suggère la distinction qu'il fait ailleurs entre «sympathie» et «engagement» (cf. Sen, 1977).

13 On relèvera que dans cette perspective, contrairement à la perspective traditionnelle, il n'y a pas de sens à s'interroger sur la rationalité d'un unique choix isolé : seuls deux choix (ou plus) peuvent être rationnels. 
14 De manière générale, il n'y a pas consensus sur le contenu exact de la partie conceptuelle de son article.

15 Le texte de 1993 montre que Sen entend cette thèse au sens fort : après la partie conceptuelle (2004 [1993], sections 1-3) qui nous occupe, joignant le geste à la parole, il ré-établit dans une partie formelle (ibid., sections 4-8) un résultat central de la théorie du choix social en n'imposant plus à la fonction de choix pertinente aucune propriété de ce type.

16 Il vaut la peine d'expliciter l'implication en jeu : Sen veut établir que la violation de la correspondance interne spécifiée par la propriété $\alpha$ n'implique pas l'incohérence interne, c'est-à-dire de manière équivalente, que la cohérence interne n'implique pas le respect de la correspondance interne spécifiée par la propriété $\alpha$. Il ne s'exprime pas sur l'implication converse, dont la négation ferait une thèse d'allure plus paradoxale encore.

17 Et d'autres propriétés encore si l'on se place, comme Sen semble ici le faire, dans le cas particulier d'une «fonction» de choix dont les ensembles choisis sont des singletons. Sur cette question, cf. Sen, 1971, section 7.

18 Pour ne pas toucher au sujet de la thèse négative telle que nous la comprenons, ces aspects n'en renvoient pas moins à des questions d'un intérêt indéniable pour la tradition réflexive globale sur les préférences révélées. Cela posé, s'agissant des questions d'observabilité en particulier, on ne saurait assez souligner que les questions de rationalité qui nous occupent ici ne s'y réduisent pas : en rigueur, les deux ensembles sont même disjoints.

19 Cette description peut obscurcir la discussion. Elle est censée éclairer (1), mais (2) aussi semble appeler une description en termes de «rejet» (comme nous allons l'expliquer : rejet d'un menu et non plus d'une option). Cela posé, précisons que l'intérêt de Sen pour le «choix libre», la liberté dessinée par un ensemble de choix donné et $\mathrm{d}$ 'autres thèmes connexes dépasse largement le cadre du contre-exemple : cf. par exemple Sen, 1985.

20 L'idée de considérer une fonction de choix définie sur des menus de menus a été diffusée, notamment depuis le travail pionnier de Kreps (1979) dans un contexte plus déterminé.

21 De ce point de vue, malgré ses défauts, l'exemple de l'ascète sourcilleux a tout de même cet avantage sur l'exemple du lecteur libéral de ramener la dimension stratégique du «choix libre» à la portion congrue : même ermite, c'est-à-dire (admettons) hors de toute interaction, un ascète sourcilleux pourrait tout à fait vérifier (2).

22 Pour clarifier les enjeux de l'exemple, nous nous écartons en effet quelque peu de la version originale.

23 De cette incertitude, il n'avait pas nécessairement conscience (le concept anglais le plus juste, qui a fait l'objet d'une élaboration technique, serait : «awareness»). Il y a d'ailleurs sur ce point une asymétrie subtile entre le cas du gourmet et celui du mondain. Le gourmet se sent d'emblée incertain quant à la qualité du restaurant et le menu le plus large lève son incertitude. Le mondain ne se sent pas nécessairement incertain d'emblée quant au caractère de son hôte potentiel, mais le menu le plus large lui rappelle qu'en fait il le connaît mal (le cas illustre d'ailleurs très bien 
la thèse suivant laquelle le degré zéro de l'incertitude naturelle est une limite jamais atteinte).

24 La fonction de choix du décideur risquerait autrement d'être mal définie, comme l'illustre une variation sur le cas du gourmet. Admettons que w symbolise «manger du fromage». Présentant au décideur, dans cet ordre, $\{x, y\},\{x, y, z\},\{x, y, z, w\}$, on pourrait avoir (2) $\{\mathrm{y}\}=\mathrm{C}(\{\mathrm{x}, \mathrm{y}\}),(1)\{\mathrm{x}\}=\mathrm{C}(\{\mathrm{x}, \mathrm{y}, \mathrm{z}\})$, mais aussi (6) $\{\mathrm{y}\}=$ $\mathrm{C}(\{\mathrm{x}, \mathrm{y}, \mathrm{z}, \mathrm{w}\}) \mathrm{si}$, comprenant avec $\mathrm{w}$ que le restaurant n'est pas minimalement casher (il propose à la fois de la viande et des laitages), un gourmet suspicieux et observant choisissait alors du poisson. Lui accordant la possibilité de réviser ses choix s'il le souhaite, on aurait alors (2), puis (5), puis à nouveau (2). À notre sens, la possibilité de telles variations incite éloquemment à considérer une fonction de choix stabilisée (étant donné les enjeux de l'exemple, cela revient en substance à assurer la stabilité, d'un menu à un autre, des croyances implicites du décideur).

25 La critique est simple, mais elle a un prix. Elle doit présupposer qu'il y a une seule vraie situation de choix dont les différents menus aident, au besoin, à cerner les caractéristiques; or, on pourrait aussi estimer que chaque menu constitue une situation de choix hypothétique à part entière (et dans ce cas, un décideur pourrait maintenir ((1) et (2))). Cela dit, la critique de Sen n'a pas intérêt à emprunter cette voie : elle est plus forte, si elle s'entend à l'état du monde constant, sans quoi l'on pourrait arguer de la variation, pour refuser de l'entendre.

26 Bien sûr, la difficulté pourra cependant ressurgir dès qu'un nouveau type de fruit sera ajouté au panier.

27 Bien sûr, la référence de $\mathrm{t}^{2}$ (celle de la description : «la part de gâteau juste en-dessous de la plus grosse part disponible») varie de menu à menu : inscrivant ceux-ci en indice, on a par exemple : $\mathrm{t}^{2}\{\mathrm{x} 0, \mathrm{x} 1\} \neq \mathrm{t}^{2}\{\mathrm{x} 0, \mathrm{x} 1, \mathrm{x} 2\}$.

28 Suivant une opposition philosophique reçue, on dira qu'on passe en fait d'une approche «extensionnelle» à une approche «intensionnelle». Sen paraît sceptique quant à la pertinence de la seconde approche dans notre contexte théorique : «quand des conditions inter-menus sont en jeu, il ne sert en général à rien d'invoquer une définition «intentionnelle» [sic] des alternatives» (2004 [1993], p. 131). On peut ne pas céder à ce scepticisme, en tout cas dans la mesure où l'intensionnel est extensionnalisable si l'on se donne un espace de propriétés (un travail en cours de Dietrich et List (mss) adapte justement les conditions inter-menus à un tel cadre, au grain plus fin).

29 Celle-ci peut être considérée comme une faiblesse des exemples : elle laisse espérer qu'en se cantonnant aux contextes non-stratégiques, on pourra échapper aux complications des contextes stratégiques. À cela Sen opposerait sans doute que le degré zéro de l'interaction (et de l'incertitude stratégique) est une limite jamais atteinte. Cela correspond en tout cas à une interprétation simple d'une des thèses de son texte de 1973 : «fondamentalement, pour cet animal social [qu'est l'homme], tout acte de choix est un acte social» (1973, p. 253).

30 Nous tirons ainsi des leçons simples d'un «jeu de la mangue» que Sen étudie subtilement ailleurs (2004 [1997], p. 180-181). 
31 Il y a là une violation d'une forme de «conséquentialisme» qu'Hammond a isolée (1988, section 4) en analysant sous forme extensive le choix dans le risque et l'incertain : à menu identique, le choix varie simplement en fonction de la place du menu dans l'arbre de décision. Sen estime que certaines de ces violations peuvent tout à fait être rationnellement défendues (en substance, c'est justement pour défendre cette possibilité qu'il invite dans son œuvre à distinguer entre «conséquence au sens restreint» et «conséquence au sens large» [2004 [1997], p. 159]).

32 Raffinement de la thèse négative quant à son esprit, elle n'en est pas moins une négation de sa lettre.

33 Cette proposition correspond (elle se ramène même fondamentalement) à une lecture de la littérature spécialisée. On peut noter en particulier que le modèle de Bhattacharyya et al. (2011, section 4), l'un des plus généraux de la littérature, procède justement en deux temps : d'abord construire un ensemble d'objets plus complexes que les options d'origine (grâce à une certaine opération, la manière dont un décideur cohérent se représente les options est intégrée), puis appliquer à ces objets non classiques les propriétés, elles classiques, des fonctions de choix, comme la propriété $\alpha$. Bien entendu, Sen connait les axes de développement de la littérature spécialisée; seulement, il n'en adopte pas la même lecture.

34 Une thèse plus forte serait que cette information ne relève que de la préférence (et non pas aussi, par exemple, de la croyance). Même si ses exemples peuvent lui sembler favorables, Sen ne revendique pas cette thèse.

35 S'il nous semble mal soutenir la thèse négative, le cas nous semble bien illustrer la thèse positive : il recouvre en fait une révision du choix qui dissout l'objection à la propriété $\alpha$, mais rend visible le travail de la préférence.

36 Il vaut la peine de préciser ce point en regard des déclarations méthodologiques citées pour commencer. On pourrait tout à fait, face à nos cas énigmatiques, avancer une théorie du choix qui ne se réfère pas à la préférence. Mais, parce qu'elle conclura à mauvais escient à une incohérence, cette théorie sera mauvaise.

37 Je suis redevable à Philippe Mongin de ses nombreux conseils. Je remercie aussi Mikaël Cozic et Philippe Lusson de leur aide, Denis Bonnay et Frantz Dietrich de leurs commentaires, ainsi que deux rapporteurs anonymes de leurs critiques.

\section{Références bibliographiques}

Arrow, Kenneth J.

1959 «Rational Choice Functions and Orderings», Economica, vol. 26, n 102 , p. 121-127.

Bhattacharyya, Aditi, Prasanta K. Pattanaik et Yongsheng Xu

2011 «Choice, Internal Consistency, and Rationality», Economics and Philosophy, vol. 27, n 2, p. 123-149.

Bossert, Walter et Kotaro Suzumura

2011 «Rationality, External Norms and the Epistemic Value of Menus», Social Choice and Welfare, vol. 37, no 4, p. 729-741.

Dietrich, Frantz et Christian List

mss «Reason-Based Rationalization» (version de travail). 
Gul, Faruk et Wolfgang Pesendorfer

2008 «The Case for Mindless Economics», dans Andrew Caplin et Andrew Schotter, dir., The Foundations of Positive and Normative Economics, Oxford, Oxford University Press, p. 3-40.

Hammond, Peter J.

1988 «Consequentialist Foundations for Expected Utility», Theory and Decision, vol. 25, $\mathrm{n}^{\circ}$ 1, p. 25-78.

Hausman, Daniel M.

2000 «Revealed Preference, Belief and Game Theory», Economics and Philosophy, vol. 16, n 1, p. 99-115.

Houthakker, Hendrik S.

1950 «Revealed Preference and the Utility Function», Economica, vol. 17, $n^{\circ} 66$, p. 159-174.

Kreps, David M.

1979 «A Representation Theorem for "Preference for Flexibility"», Econometrica, vol. 47, $n^{\circ} 3$, p. 565-577.

Little, Ian M.D.

1949 «A Reformulation of the Theory of Consumer's Behaviour», Oxford Economic Papers, New Series, vol. 1, no 1, p. 90-99.

Luce, R. Duncan et Howard Raiffa

1957 Games and Decisions, New York (NY), John Wiley \& Sons.

MasCollel, Andreu, Michael D. Whinston et Jerry R. Green,

1995 Micro-Economic Theory, Oxford, Oxford University Press.

Mongin, Philippe

2000 «Les préférences révélées et la formation de la théorie du consommateur», Revue économique, vol. 51, no 5, p. 1125-1152.

2011 «La théorie de la décision et la psychologie du sens commun», Social Science Information, vol. 50, $\mathrm{n}^{\text {os }} 3-4$, p. 351-374.

Pettit, Philip

1991 «Decision Theory and Folk Psychology», dans Michael Bacharach et Susan Hurley, dir., Foundations of Decision Theory, Oxford, Blackwell, p. $147-175$.

Samuelson, Paul A.

1938 «A Note on the Pure Theory of Consumer Behaviour», Economica, vol. $5, \mathrm{n}^{\circ} 17$, p. 61-71.

1950 «The Problem of Integrability in Utility Theory», Economica, vol. 17, $n^{\circ} 68$, p. $355-385$.

Sen, Amartya K.

1971 «Choice Functions and Revealed Preference», The Review of Economic Studies, vol. 38, n 3, p. 307-317.

1973 «Behaviour and the Concept of Preference», Economica, vol. 40, n 159, p. 241-259.

1977 «Rational Fools: A Critique of the Behavioral Foundations of Economic Theory», Philosophy and Public Affairs, vol. 6, n 4, p. 317-344. 
1985 Commodities and Capabilities, Amsterdam, Elsevier.

2004 «Internal Consistency of Choice» [1993], dans Rationality and Freedom, Cambridge, Belknap Press, p. 121-157.

2004 «Maximization and the Act of Choice» [1997], dans Rationality and Freedom, Cambridge, Belknap Press, p. 158-205.

Wong, Stanley

2006 The Foundations of Paul Samuelson's Revealed Preference Theory [1978], New York (NY), Routledge. 